\title{
Integration von Künstlicher Intelligenz in die Fabriksteuerung
}

Norbert Gronau

\begin{abstract}
Mit der zunehmenden Verfügbarkeit von loT-Devices und einer deutlich stärkeren Vernetzung der Fertigung mit Internet-Technologien rückt auch eine Verbesserung der Fabriksteuerung durch den Einsatz Künstlicher Intelligenz (KI) in den Vordergrund. Dieser Beitrag beschreibt am Beispiel einer variantenreichen Serienfertigung, welche Schritte zu gehen sind, um mit KI die Fabriksteuerung zu verbessern.
\end{abstract}

Künstliche Intellligenz kann zunächst definiert werden als die Entwicklung von Informationssystemen, die in der Lage sind, Aufgaben zu erledigen, die normalerweise menschliche Intelligenz erfordern. Zu den Komponenten, die eine $\mathrm{KI}$ ausmachen, zählen normalerweise eine geeignete Wissensrepräsentation, Schlussfolgerungsmechanismen (Reasoning) und vor allem in neuerer Zeit, Fähigkeiten zur selbständigen Erweiterung der Wissensbasis durch Lernen. Dafür ist insbesondere das Deep Learning entwickelt worden, mit dem es möglich wird, durch einen mehrschichtigen Aufbau von Neuronalen Netzwerken (Bild 1), die Merkmale klassifizieren, ein Lernen ohne die Notwendigkeit der menschlichen Überwachung zu realisieren.

\section{Ziel des Kl-Einsatzes in der Fabriksteuerung}

In der variantenreichen Serienfertigung verfolgt die Fabriksteuerung das Ziel, eine möglichst gute Reihenfolge der Auftragsbearbeitung zu erreichen. Die unterschiedlichen
Varianten weisen eine stark voneinander abweichende Durchlaufzeit auf, deren unreflektierte Einplanung zu ungleichmäßigen Takten und unterdurchschnittlich ausgelastetem Montagepersonal führen.

Durch den Einsatz von KI als Ergänzung der bisher eingesetzten Methoden sollen dabei nun folgende Verbesserungen erreicht werden:

- Verkürzung der Durchlaufzeiten durch Reihenfolgeplanung in Abhängigkeit von der variantenspezifischen Bearbeitungszeit

- Berücksichtigung von Abhängigkeiten bei hoher Variantenvielfalt

- Optimierung der Zusammenstellung von Reihenfolgen durch geeignete Klassifizierung und Prognosefähigkeit

- Optimierte Zusammenstellung von Varianten in Abhängigkeit von unterschiedlichen Produktionsszenarien

- Hohe Planungsqualität bzgl. der Reihenfolgenzusammenstellung durch Anwendung von KI-Verfahren

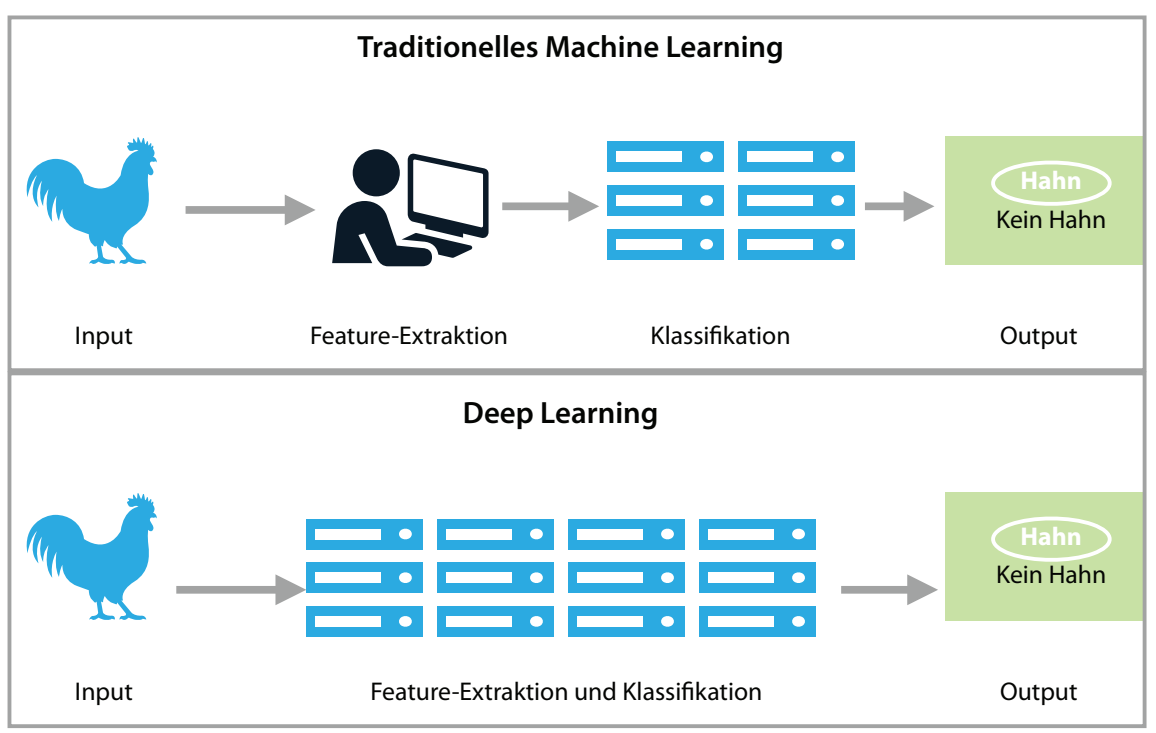

Bild 1: Unterschied zwischen traditionellem Machine Learning und Deep Learning [1]
Komponenten von Künstlicher Intelligenz

Potenziale von Künstlicher Intelligenz in der Fabriksteuerung

Zielführende Schritte bei einem KI-Projekt in der variantenreichen Serienfertigung 


\section{Konkrete Problemstellung}

Die Produktpalette des betrachteten Unternehmens ist durch hohe Variantenvielfalt gekennzeichnet (ca. 1.300 Ausstattungsmerkmale, kombiniert mit 10 Bauvarianten). Für die gesamte Planung und Steuerung der Produktion ist ein Leitstand verantwortlich. Dessen zu beherrschende Planungsaufgabe erstreckt sich von der Koordination sämtlicher Gewerke (Umformtechnik, Lackiererei, Montage, Logistik) bis hin zur Eintaktung des Warenausgangs. Besonderer Fokus liegt auf der Gestaltung einer optimalen Reihenfolge der zu produzierenden Varianten hinsichtlich Durchlaufzeit und Prognose des Ressourcenbedarfs (Mitarbeiter, Schnittstellen zu internen und externen Lieferanten im just in time und just in sequence-Prinzip). Dies führt zu einer hohen Komplexität der Planung. Mit Hilfe von $\mathrm{KI}$ soll eine intelligente Fabriksteuerung realisiert werden, die auf Basis der aktuellen Situation sowie kausaler Zusammenhänge aus historischen Daten Vorschläge für eine optimale Steuerung der Fabrik bereitstellt und es damit dem Leitstand ermöglicht, verbesserte Entscheidungen zu treffen.

\section{Vorgehen zur Integration von $\mathrm{KI}$ in die Fabriksteuerung}

Die oben skizzierten Ziele werden mit Hilfe von vier KI-Komponenten realisiert, mit Hilfe derer die für die Lösung der Aufgabenstellung relevanten Modelltypen bereitgestellt werden (Bild 2).

Das Projekt wurde gemäß Bild 2 in vier Teilprojekte aufgeteilt, die einzeln bereits jeweils einen Nutzen für den Auftraggeber liefern. In der Kombination der vier Teilprojekte wird die Gesamtzielstellung erfüllt.

\section{Bild 2: Strukturierung des Projektes}

\section{Teilprojekt 1: Klassifikation}

Dieses Teilprojekt liefert ein Modell für eine geeignete Klassifikation der unterschiedlichen Varianten nach geeigneten Zieldimensionen. Ziel ist es, die Varianten hinsichtlich der Zielerreichung in verschiedenen Zieldimension zu bewerten. Diese ist als Grundlage für die folgenden Teilprojekte erforderlich. So werden z. B. für die Durchlaufzeit der Variante die Kategorien "kurz", "mittel", oder "lang" vorgeschlagen. Entsprechend wird für weitere Zieldimensionen verfahren.

Um diese Aufgabe zu lösen, wird die Relevanz der einzelnen Variantenmerkmale für Durchlaufzeitabweichungen untersucht, indem beispielsweise analysiert wird, welche Merkmale an welcher Arbeitsstationen zu verlängerten Taktzeiten führen. Diese Analysen werden sowohl explorativ mit dem Fokus auf dem Erkennen von Clustern sowie exploitativ mit dem Fokus auf dem Einteilen der Variantenmerkmale anhand übereinstimmender Merkmale (Klassifikation) durchgeführt. Als Ergebnis dieses Teilprojektes wird eine verbesserte und erfolgswirksame Charakterisierung der Variantenmerkmale erreicht.

Der Nutzen für den Auftraggeber nach Realisierung dieses Teilprojektes besteht darin, dass Produktionsabläufe verbessert werden können, indem sich die Zusammenstellung von Variantenmerkmalsfolgen an dieser statischen Charakterisierung orientiert. Weitere Iterationen des oben skizzierten Vorgehens verbessern die Güte der Klassifikation und optimieren so die Wirksamkeit der Ergebnisse.

\section{Teilprojekt 2: Prognose}

Dieses Teilprojekt hat die Aufgabe, Modelle für eine geeignete Prognose der Varianten und Produktionsszenarien nach verschiedenen und zu wählenden Zieldimensionen zu erzeugen. Es soll die Realisierung der Prognosefähigkeit des Produktionsverlaufs in Abhängigkeit von Variantenmerkmalsfolgen erreicht werden.

Um dies zu bewerkstelligen, wird die dynamische Relevanz der Variantenmerkmale im Produktionsverlauf untersucht. Es wird dabei zum Beispiel analysiert, welche Merkmale an welcher Produktionsstation zu erhöhten Taktzeiten führen. Da- 
rauf kann dann eine Prognose von erwarteter Stillstandszeit, Fehleranzahl und Ausfallquote aufgebaut werden. Anschließend wird die Prognosefähigkeit auf weitere Zieldimensionen ausgedehnt.

Ergebnis dieses Teilprojektes ist eine verbesserte dynamische und erfolgswirksame Charakterisierung der Variantenmerkmale im Produktionsverlauf. Es wird ermöglicht, Produktionsabläufe zu verbessern, indem durch die dynamische Charakterisierung die geeignete Zusammenstellung von Variantenmerkmalsfolgen prognostiziert werden kann. Weitere Iterationen verbessern auch in diesem Teilprojekt die Prognosegüte und damit die Erzeugung eines optimalen Produktionsplans.

\section{Teilprojekt 3: Optimierung}

In diesem Teilprojekt wird die Zusammenstellung von Fahrzeugvarianten entsprechend betrachteter Produktionsszenarien nach verschiedenen und zu wählenden Zieldimensionen optimiert. Dazu werden die Variantenmerkmalsfolgen im Gesamtproduktionskontext integrativ analysiert. Beispielsweise wird untersucht, wie Ergebnisse diverser KI-Komponenten (über den Fokus von Teilprojekt 1 und 2 hinaus) zu verbesserten Produktionsdurchläufen verbunden werden können. Zu optimierende Größen in diesem Teilprojekt sind die erwartete Stillstandszeit, Fehleranzahl und Ausfallquote. Weitere Optimierungsgrößen werden überprüft.

Ergebnis dieses Teilprojektes ist außerdem die Möglichkeit, ganzheitliche Handlungsempfehlungen für die Optimierung von Produktionsdurchläufen zu generieren. Im Fokus steht dabei nun eine globale Optimierung des betrachteten Produktionsablaufs. Dies befähigt zu Sensitivitätsanalysen und Plausibilitätstests von einzelnen Szenarien, z. B. hinsichtlich der Reihenfolgeplanung oder der Wirksamkeit gewählter Maßnahmen zur Verbesserung der produktionswirtschaftlichen Zielgrößen.

Für den Auftraggeber ist als erheblicher Nutzen anzusehen, dass er seinen gesamten Produktionsbereich hinsichtlich der gewählten Zieldimension verbessern und unter Einbeziehung der Prognosen eine robustere Planung der Produktion umzusetzen vermag.

\section{Teilprojekt 4: Integration}

Dieses Teilprojekt basiert auf einer Betrachtung vorhandener Vorgehensweisen für die Planung beim Auftraggeber und harmonisiert diese mit KI-Komponenten. Ziel ist es, die Produktionsplanung anhand der Zusammenstellung von Produktvarianten nach verschiedenen Produktionsszenarien und zu wählenden Zieldimensionen zu optimieren. Dazu wird untersucht, wie $\mathrm{KI}$-Planungsprozesse mit existierenden Planungsprozessen der Ausgangssituation verbunden und effizient gestaltet werden können. Dabei werden weitere state-of-the-artAnsätze der Reihenfolgeplanung ebenfalls auf ihre Integrierbarkeit hin betrachtet, etwa ein Ansatz zur systematischen Durchwandern des Lösungsraumes [2]. Unter anderem wird geprüft, ob die Ausdehnung der Planung auf weitere Bereiche oder der Einsatz weiterer Reihenfolgeplanungsmechanismen zu verringerten Taktzeiten führen. Planungshorizont ist dabei die monatliche, wöchentliche oder tägliche Auslastung der Produktion. Auch hier werden mehrere Iterationen durchgeführt, um die Planungs-, bzw. Reihenfolgegüte zu verbessern. Insgesamt wird durch das Projekt eine einheitliche Planungsgrundlage geschaffen, die unterschiedliche teilweise bereits vorhandene Planungsansätze mit den neu geschaffenen $\mathrm{KI}$-Komponenten verbindet.

Nutzen: Es wird ermöglicht, Produktionsabläufe zu verbessern, indem die Planung von der Ausstattungscodefolgen-Zusammenstellung an effizienten Ansätzen orientiert wird. Somit verbessert sich die Gesamtproduktion hinsichtlich der gewählten Zieldimension.

Dieses Projekt zeigt, dass dem ergebniswirksamen Einsatz von KI-Komponenten zunächst eine Klassifikation der verfügbaren Daten vorausgehen muss. Eine weitere Herausforderung stellt die Integration der bereits genutzten Planungsmechanismen und die gesamthafte Optimierung mehrerer produktionswirtschaftlicher Ziele dar. Wenn diese allerdings erreicht sind, steht einer weiteren Optimierung durch Einsatz von cyber-physischen Systemen [3] nichts mehr im Wege!

Der Autor dankt Dr. Sander Lass, Dr. Edzard Weber und Marcus Grum für ihre wertvollen Hinweise.

Schlüsselwörter:

Künstliche Intelligenz, Fabriksteuerung, Deep Learning, Serienfertigung, Varianten
Literatur

[1] https://www.gurug9. com/images/tensorflow/083018_0454_MachineLear5.png (Letzter Abruf 11.10.2019)

[2] Weber, E., Tiefenbacher, A. Gronau, N.: Need für Standardization and Systematization of Test Data for Job-Shop Scheduling. Data 2019, 4 (1), 32; https://doi.org.10.3390/ data4010032 (Letzter Abruf 11.10.2019)

[3] Lass, S.: Nutzenvalidierung cyber-physischer Systeme in komplexen Fabrikumgebungen. Berlin 2017
Integration of Artificial Intelligence into Factory Control

With the increasing availability of IoT devices and a sig nificantly stronger networking of production with Internet technologies, an improvement in factory control through the use of artificial intelligence is also moving to the fore. This article describes the steps that need to be taken to improve factory control with Al using the example of a variant-rich series production.

Keywords:

Artificial Intellgence, Factory Contol, Deep Learning,

Serial Production, Variants 